\title{
BINDING ENERGY OF MUONIUM HYDRIDE
}

\author{
M. SUFFCZYŃSKI \\ Institute of Physics, Polish Academy of Sciences \\ Al. Lotników 32/46, 02-668 Warszawa, Poland \\ AND L. WOLNIEWICZ \\ Institute of Physics, Nicolas Copernicus University \\ Grudziądzka 5, 87-100 Toruń, Poland \\ (Received September 4, 1992)
}

\begin{abstract}
Binding energy of muonium hydride is calculated variationally with wave function dependent exponentially on three interparticle distances. The lower bound for the dissociation energy into the hydrogen atom and muonium is obtained as $3.853 \mathrm{eV}$. Expectation values of the interparticle distances are also calculated.

PACS numbers: $36.10 . \mathrm{Gv}, 71.35 .+\mathrm{z}$
\end{abstract}

The muonium hydride, a system consisting of the muonium atom and a hydrogen atom is, after the positronium hydride, an example of a four-particle neutral system. Its ground state has the same quantum numbers as the positronium hydride $[1,2]$. The difference between the two systems is in the mass of the positron and that of the positive muon. The proton-positron mass ratio is $m_{\mathrm{p}} / m_{\mathrm{e}}=1836.152701$, the proton-muon mass ratio $m_{\mathrm{p}} / m_{\mu}=8.8802444$ [3].

The muon spin precession in magnetic field is well measured in solids and in liquids [4-10]. Muonium is observed by means of its triplet state precession [5]. Muonium may become incorporated in a variety of molecules and chemical consequences of replacement of hydrogen by muonium in a molecule can be observed [6]. Therefore, consideration of muonium bound to a neutral atom with immobile nucleus can be investigated also as a system of considerable interest. We calculated the ground state of muonium hydride with a variational wave function, symmetrized in the coordinates of the two electrons of the molecule, as described previously [11-13]. The wave function was a linear combination of monomials in four interparticle distances with an overall exponential factor dependent on the three lepton distances from the most massive particle. The most massive particle was a proton in muonium hydride, $\mathrm{HMu}$, a deuteron with mass $m_{\mathrm{d}}=3670.483014 m_{\mathrm{e}}$ in DMu [3], a triton with mass $m_{\mathrm{t}}=5496.918 m_{\mathrm{e}}$ in TMu [14, 15], and an immobile proton in $\mathrm{H}_{\infty} \mathrm{Mu}$. We used the corresponding reduced masses $\mu_{\mathrm{r}}$ of the muon as shown in Table I. For the triton mass we used the larger of the two quoted [14-17] 


\section{TABLE I}

Computed ground state energy $E$ in atomic units $(1$ a.u. $=27.2113961 \mathrm{eV}[3])$ and dissociation energy $D$ (in $\mathrm{eV}$ ) of muonium hydride system, with muon reduced mass $\mu_{\mathrm{r}}$ given in the second column in units of electron mass.

\begin{tabular}{c|c|c|c}
\hline \hline & $\mu_{\mathrm{r}}$ & $-E$ & $D$ \\
\hline $\mathrm{HMu}$ & 185.841 & 1.1389 & 3.853 \\
$\mathrm{DMu}$ & 195.742 & 1.1395 & 3.865 \\
$\mathrm{TMu}$ & 199.273 & 1.1397 & 3.869 \\
$\mathrm{H}_{\infty} \mathrm{Mu}$ & 206.768262 & 1.1401 & 3.877
\end{tabular}

values but this does not affect the value of the reduced mass within the digits retained by us. The values of the three nonlinear parameters in the exponential factor of the variational wave function were determined by extrapolation from Ref. [13] and then by successive trials.

The ground state energy was computed with a wave function comprising all monomials with the interparticle distance powers whose sum $\omega$ did not exceed 7 , which gives 330 linear terms. Table I gives the computed ground state energy $E$ of the muonic molecule, in atomic units, and the energy $D$, in $\mathrm{eV}$, of dissociation into the hydrogen atom and muonium atom. For HMu we have got the lower bound of the dissociation energy $D=0.14159$ a.u. $=3.853 \mathrm{eV}$. In Table II the calculated

TABLE II

Expectation values of the interparticle distances in the muonium hydride in units of the electron Bohr radius $a_{\mathrm{B}}=0.529177249 \times 10^{-8} \mathrm{~cm}[3]$.

\begin{tabular}{c|c|c|c|c}
\hline \hline \multicolumn{5}{c}{ Expectation values of the first inverse powers of distances } \\
\hline & $\left\langle r_{1}^{-1}\right\rangle^{-1}$ & $\left\langle r_{12}^{-1}\right\rangle^{-1}$ & $\left\langle r_{13}^{-1}\right\rangle^{-1}$ & $\left\langle r_{3}^{-1}\right\rangle^{-1}$ \\
\hline $\mathrm{HMu}$ & 1.12 & 1.78 & 1.16 & 1.47 \\
$\mathrm{H}_{\infty} \mathrm{Mu}$ & 1.11 & 1.78 & 1.16 & 1.46 \\
\hline
\end{tabular}

Expectation values of the first powers of distances

\begin{tabular}{c|c|c|c|c}
\hline & $\left\langle r_{1}\right\rangle$ & $\left\langle r_{12}\right\rangle$ & $\left\langle r_{13}\right\rangle$ & $\left\langle r_{3}\right\rangle$ \\
\hline $\mathrm{HMu}$ & 1.60 & 2.28 & 1.67 & 1.53 \\
$\mathrm{H}_{\infty} \mathrm{Mu}$ & 1.60 & 2.28 & 1.67 & 1.53
\end{tabular}

expectation values of the interparticle distances are displayed. The $r_{1}$ and $r_{2}$ are the proton-electron distances, $r_{12}$ is the distance between the two electrons, $r_{13}$ is the electron-muon and $r_{3}$ - the electron-proton distance. Expectation values 
of the inverse distances $\left\langle r^{-1}\right\rangle$ were computed in the calculation of the Coulomb potential energy of the system, with the 330-term wave function with optimized nonlinear parameters. Expectation values of the first positive power of distances $\langle r\rangle$ were computed with $\omega \leq 6$, i.e. with 210 -term wave function. The results show that the muonium hydride is in its size similar to the neutral hydrogen molecule $[13,18]$ rather than to a positronium hydride [2]. The computations were performed in double precision on SPARC 2 computer, and an improvement of precision is desirable. The reservation has to be added that the trial wave function used to describe the muonium hydride may not be the best and a better wave function may give larger value of the dissociation energy and presumably smaller interparticle distances.

\section{References}

[1] P.B. Navin, D.M. Schrader, C.F. Lebeda, Phys. Rev. A 9, 2248 (1974).

[2] Y.K. Ho, Phys. Rev. A 34, 609 (1986).

[3] E.R. Cohen, B.N. Taylor, Rev. Mod. Phys. 59, 1121 (1987).

[4] A. Weidinger, Ch. Niedermayer, A. Golnik, R. Simon, E. Recknagel, J.J. Budnick, B. Chamberland, C. Baines, Phys. Rev. Lett. 62, 102 (1989).

[5] E. Roduner, Prog. Reaction Kinetics 14, 1 (1986).

[6] S.F.J. Cox, J. Phys. C, Solid State Phys. 20, 3187 (1987).

[7] B.D. Patterson, Rev. Mod. Phys. 60, 69 (1988).

[8] R.F. Kiefl, J.W. Schneider, A. MacFarlane, K. Chow, T.L. Duty, T.L. Estle, B. Hitti, R.L. Lichti, E.J. Ansaldo, C. Schwab, P.W. Percival, G. Wei, S. Wlodek, K. Kojima, W.J. Romanow, J.P. McCauley Jr., N. Coustel, J.E. Fischer, A.B. Smith III, Phys. Rev. Lett. 68, 1347, 2708(E) (1992).

[9] J.W. Schneider, R.F. Kiefl, K. Chow, S.F.J. Cox, S.A. Dodds, R.C. DuVarney, T.L. Estle, R. Kadono, S.R. Kreitzman, R.L. Lichti, C. Schwab, Phys. Rev. Lett. 68, 3196 (1992).

[10] M. Casarin, G. Granozzi, E. Tondello, A. Vittadini, Chem. Phys. 156, 385 (1991), 159, 365 (1992).

[11] B.A. Page, P.A. Fraser, J. Phys. B, At. Mol. Phys. 7, L389 (1974).

[12] B. Stebe, G. Munschy, Solid State Commun. 35, 557 (1980).

[13] M. Suffczyński, L. Wolniewicz, Phys. Rev. B 40, 6250 (1989).

[14] K. Szalewicz, H.J. Monkhorst, W. Kolos, A. Scrinzi, Phys. Rev. A 36, 5494 (1987).

[15] A. Scrinzi, H.J. Monkhorst, S.A. Alexander, Phys. Rev. A 38, 4859 (1988).

[16] K. Szalewicz, B. Jeziorski, A. Scrinzi, Y. Zhao, R. Moszynski, W. Kolos, P. Froelich, H.J. Monkhorst, A. Velenik, Phys. Rev. A 42, 3768 (1990).

[17] B. Jeziorski, K. Szalewicz, Phys. Lett. A 152, 240 (1991).

[18] L. Wolniewicz, J. Chem. Phys. 45, 515 (1966). 